\title{
Periostin in fibrillogenesis for tissue regeneration: periostin actions inside and outside the cell
}

\author{
Akira Kudo
}

Received: 12 July 2011 / Revised: 19 July 2011/Accepted: 19 July 2011/Published online: 11 August 2011

(C) The Author(s) 2011. This article is published with open access at Springerlink.com

\begin{abstract}
More than 10 years have passed since the naming of periostin derived from its expression sites in the periosteum and periodontal ligament. Following this finding, we have accumulated more data on the expression patterns of periostin, and, finally, with the generation of periostin-deficient mice, have revealed functions of periostin in the regeneration of tissues in bone, tooth, heart, and skin, and its action in cancer invasion. Since periostin is a matricellular protein, the first investigation of periostin function showed its enhancement of cell migration by acting outside the cell. On the other hand, recent observations have demonstrated that periostin functions in fibrillogenesis in association with extracellular matrix molecules inside the cell.
\end{abstract}

Keywords Periostin - Fibrillogenesis - Bone ·

Type I collagen · Tooth $\cdot$ Heart $\cdot$ Matricellular protein ECM

\section{Introduction}

Periostin [1], a matricellular protein, was named based on its expression in the periodontal ligament and periosteum of adult mice. This protein was expected to function in tooth and bone remodeling under mechanical stress, since both the periodontal ligament and periosteum are sites sensitive to mechanical stress by mastication and physical exercise, respectively. Such stress induces remodeling of the periodontal ligament and parietal bone. After

A. Kudo $(\square)$

Department of Biological Information,

Tokyo Institute of Technology, Yokohama 226-8501, Japan

e-mail: akudo@bio.titech.ac.jp inflammation, the appearance of TGF $\beta$ induces periostin expression. Periostin, a $90-\mathrm{kDa}$ secreted protein, has a protein structure composed of an amino-terminal EMI domain, a tandem repeat of 4 fas I domains, and a carboxyl-terminal domain including a heparin-binding site at its C-terminal end (Fig. 1); therefore, we characterized it as a member of the fasciclin I family based on these typical fas I domains. Fasciclin I is a GPI-anchored Drosophila protein containing 4 tandem fas I domains composed of about 150 amino acid residues each, which are not related to any other protein domain of known structures [2], and it functions in axon growth guidance. In humans, fas I domains are found in $\beta$ igh 3 [3] and stabilins [4] as well as in periostin [5]. Periostin and $\beta$ igh 3 are most similar, sharing uninterrupted tandem repeats of 4 fas I domains. Interestingly, mutations in fas I domains of human $\beta$ igh3 result in corneal dystrophy due to the deposition of insoluble protein aggregates in the cornea [6]. The EMI domain, which is a small module rich in cysteine residues that is found in the EMILIN family, is a site for protein-protein interaction [7].

Periostin directly interacts with type I collagen [8-10], fibronectin [11] and Notch1 [12] through its EMI domain and interacts with tenascin-C [11] and BMP-1 [13] through its fas I domains. These periostin interactions with mainly extracellular matrix (ECM) molecules firstly occur intracellularly [11]. Furthermore, periostin serves as a ligand of integrins such as $\alpha \mathrm{v} \beta 3$ and $\alpha \mathrm{v} \beta 5$ and promotes cell motility [14] by acting outside the cell. In light of these properties of protein interactions, I have classified periostin action into two major functional categories, i.e., fibrillogenesis, which occurs inside the cell, and cell migration outside the cell, involving its extracellular action.

After cloning the periostin gene, the establishment of 3 independent periostin-knockout mice provided the first 


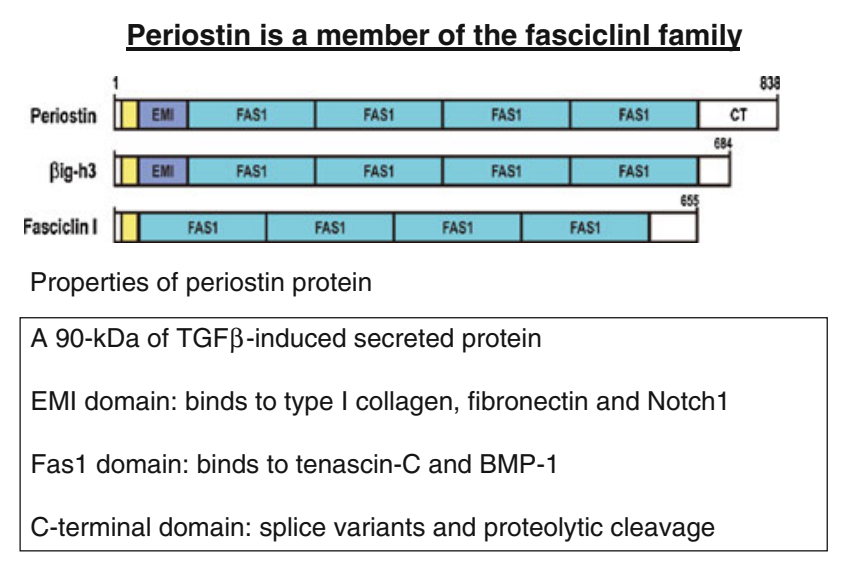

Fig. 1 Properties of periostin protein. Periostin is a 90-kDa secreted protein, and its expression is induced by TGF $\beta$. The protein structure of periostin comprises an amino-terminal EMI domain, a tandem repeat of 4 fas1 domains, and a carboxyl-terminal domain. From conservation of the typical fas 1 domains, we characterized periostin as being a member of the fasciclin I family, which includes $\beta$ igh 3 and Drosophila fasciclin I. Periostin domains have the following characteristics: the EMI domain binds to type I collagen, fibronectin, and Notch1; and the Fas I domains bind to tenascin-C and BMP-1. The $\mathrm{C}$-terminal domain gives rise to splice variants and contains proteolytic cleavage sites

insights into periostin function [8, 15-17]. These studies demonstrated that periostin functions in regeneration of tissues such as bone, tooth, and heart, and in cancer invasion and wound healing.

Several reviews on the involvement of periostin in cardiac development [18], tumorigenesis [19], and wound healing [20] have already been published. Therefore, I would like to focus on recent findings that support a new concept of periostin function, i.e., its participation in fibrillogenesis.

\section{Periostin in bone and fibrillogenesis}

From the initial stage of the periostin research, we expected to find that periostin functions in mechanical stress-induced regeneration of periosteal bone. Periostin is specifically expressed in the periosteum, which is a complex structure composed of an outer fibrous layer lending structural integrity and an inner cambium layer possessing osteogenic potential [21]. In periostin-deficient mice, cortical bone in the femur shows a decrease in area and thickness [11]. Moreover, ectopically mineralized deposits are observed in periostin $-/-$ mice following a thickening of the periosteum, which abnormal phenotype resembles periostitis. A similar phenotype was observed in tenascin-C-deficient mice, suggesting that periostin is associated with tenascin$\mathrm{C}$ to elaborate an ECM meshwork architecture that includes fibronectin and type I collagen (Fig. 2). This

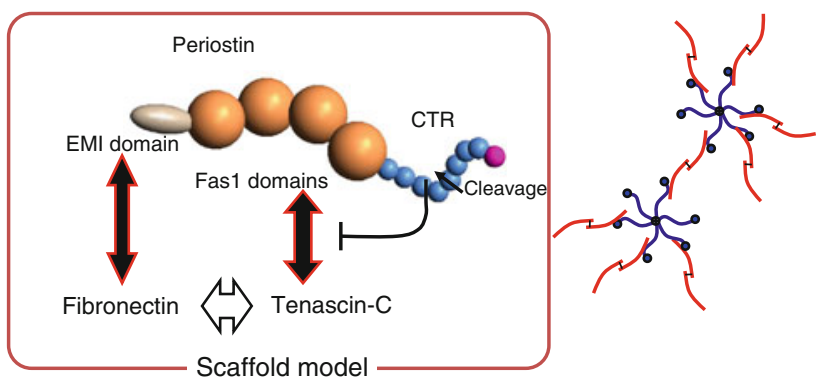

Fig. 2 Assembly between fibronectin and tenascin-C by periostin. Cleavage of the C-terminal end of perostin, including the heparinbinding site (pink), can induce association of the truncated periostin with tenascin-C via the fas I domains of periostin. Fibronectin is associated with periostin at the EMI domain of the latter, and with tenascin-c to form the specific hexabrachion structure, which behaves as a scaffold, in which tenascin-C forms a disulfide-linked hexamer (blue) that binds to the dimer form of fibronectin (red) via periostin

architecture generates a microenvironment for mechanical stress-dependent bone formation. Consistently, we observed reduced collagen cross-links for fibrillogenesis in the periosteum from periostin $-/-$ femurs. Defective cross-linkage is a common feature of periostin function reported in periostin-deficient mouse skin [10] and heart tissues after a myocardial infarction [15].

The molecular mechanism of periostin action in collagen cross-linking has been investigated, and the results showed that periostin enhances the proteolytic activation of lysyl oxidase (LOX), which is an enzyme responsible for cross-link formation, and that it is caused by interacting with BMP-1 to promote the collagen cross-linking [13] as shown in Fig. 3. In this activation of LOX, we propose the involvement of a special ECM structure based on fibronectin to which precursor LOX and periostin can bind; then, periostin stabilizes the precursor LOX to allow it to be effectively proteolyzed to generate the mature LOX. According to this scenario, periostin functions in high stiffness collagen formation with effective collagen crosslinking.

What is the molecular mechanism for generating this collagen structure? In tendon, bone, and skin, type I collagen is the major component of these fibrils. In fibrillogenesis, collagen fibril formation in cell culture is dependent on the prior assembly of fibronectin into fibrils. Therefore, collagen fibrillogenesis that occurs on the cell surface is downstream of fibronectin assembly, and it is mediated by interactions with cell-surface integrins [22]. Tenascin-C forms a typical disulfide-linked hexamer, called the hexabrachion, in which six flexible arms emanate from a central globular particle, which possibly catches and stabilizes a bifurcation of the ECM fibrils composed of fibronectin and type I collagen to underlie the extracellular meshwork architecture. Periostin and 
Fig. 3 Periostin function in collagen cross-link formation. Periostin recruits BMP-1 onto the fibronectin matrix to enhance LOX activity for collagen cross-linkage [13]

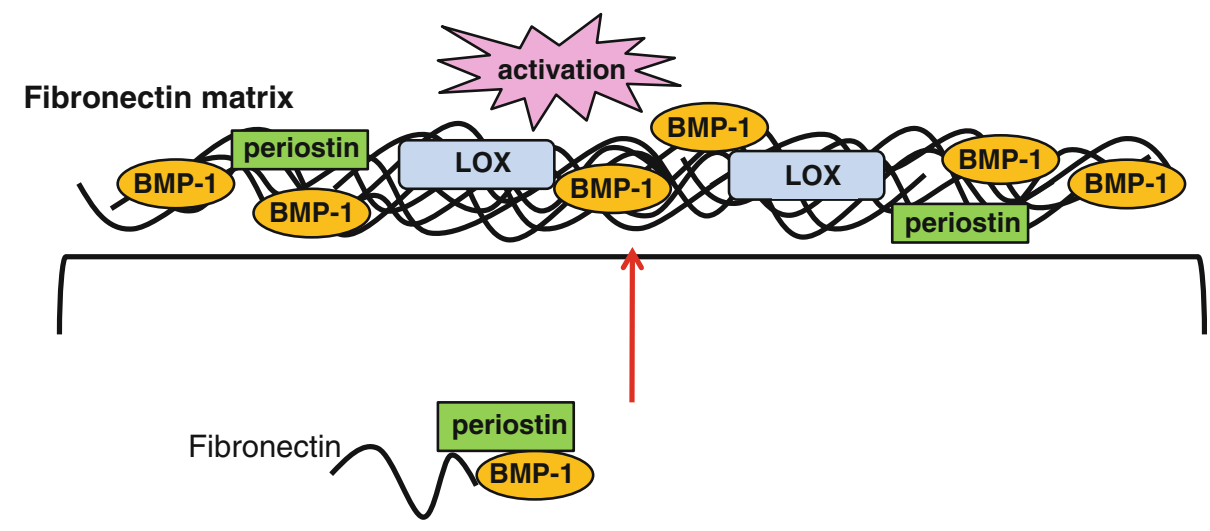

tenascin-C expression are coordinately induced by mechanical stress, with transcription of these genes being regulated by cytoskeletal actin [23], indicating that periostin-mediated incorporation of tenascin-C into the ECM architecture maintains structural homeostasis when adapting to a changing mechanical environment.

\section{Periostin in teeth}

The first observed phenotype of periostin $-/-$ mice was fragility of the teeth, mainly caused by defective periodontal ligaments [8, 16]. Based on expression analysis of periostin in embryonic teeth of the mouse mandible, the periostin protein was localized to the interface between the inner enamel epithelium and preodontoblasts as well as in the mesenchymal tissues around the cervical loop [24]. In juvenile mice at postnatal day 7 , periostin protein is restricted to the fibrous bundles in the periodontal ligament in accordance with the organization of periodontal fibers [24]. These findings suggest that periostin is involved at sites of cell-to-matrix interaction, serving as adhesive equipment for bearing mechanical forces including tooth eruption, and transducing the occlusal force that activates latent TGF $\beta$ to enhance periostin expression [25]. Consistently, periostin $-/-$ mice show defective eruption of their incisors [8] and degradation of the periodontal ligament of molars during experimental tooth movement [12]. The abnormal presence of non-digested collagen fibrils in the shear zone in the periostin $-/-$ periodontal ligament [8] is now explained by the low activity of MMPs, which are efficiently secreted following their induction by periostin [26].

\section{Periostin in mechanotransduction}

It still remains to be investigated whether periostin is directly connected with the mechanosensor. From the specific expression of periostin in the periosteum and periodontal ligament, we expected that periostin would be linked to mechanical stress. To search for this linkage, we looked for an upstream signal regulating periostin expression that would act in a transcriptional manner. Investigating the periostin promoter, we found that twist, a basic helix-loop-helix transcription factor, binds to upregulate its transcription [27]. To further demonstrate the involvement of periostin and twist in mechanical stress, we prepared an experimental model in which teeth were subjected to movement. This forced mechanical stress upregulated periostin transcription [28]. In contrast, occlusal hypofunction decreases the expression of both periostin and twist [29]. Although the up-stream signals that regulate twist remain unknown, periostin is very closely involved with the mechanosensing signal. Analysis of the periostin promoter sequences revealed predicted c-fos-binding sites, which may provide for the ectopic expression of periostin in the bone marrow of c-fos transgenic mice and in that of patients with fibrous dysplasia (FD) [30].

Specific transcription of the periostin gene in embryonic fascias of muscles that receive mechanical stress in mice provides further evidence of the involvement of periostin in mechanical sensing [31]. Another animal model using zebrafish showed that periostin is expressed in the myoseptum between muscles. This tissue senses mechanical stress and delivers that signal from one muscle to another [32]. The knock-down of periostin with morpholino antisense oligonucleotide leads to defects in myoseptum formation, a delay in the differentiation of myofibers, and disorder of the connection between myofibrils and myoseptum [32]. Similar to the myoseptum in zebrafish, tendons in mammals are a uniaxial connective tissue that functions in the transmission of forces from the musculature to the skeletal system [33]. The relationship between periostin and the mechanical integrity of tendons needs to be further investigated.

In another study involving periostin in mechanotransduction, periostin was shown to negatively regulate the 
action of sclerostin, which is a receiver of bone-related mechanical stress signals. Consequently, mechanical stimuli have no effect on the skeletal properties in periostin $-/-$ mice [34]. All these results indicate that periostin is very closely related to the mechanosensor.

\section{Periostin in heart disease}

Periostin is expressed in the heart valves throughout life from embryo to adult. However, we could not observe any significant abnormalities in the formation of heart valves, which would indicate that periostin mainly functions in heart diseases in association with tissue repair. The expression of periostin in the embryonic heart has already been reviewed by Norris et al. [10]. Therefore, I will focus here on periostin function in heart diseases.

In humans, periostin is abundantly expressed in the infarct border after a myocardial infarction. In mice, periostin expression is induced by $\operatorname{TGF} \beta$ following inflammation at the infarct border [15]. To further study these expression patterns, we assessed the regeneration of heart tissues after a myocardial infarction in periostin -/mice [15]. Consistent with the findings made by Oka et al. [17], we demonstrated that periosin functions in the migration of cardiac fibroblasts through engaging integrin $\alpha \mathrm{v} \beta 3$, which induces phosphorylation of the downstream kinase FAK (focal adhesion kinase), leading to the production of type I collagen. Thereafter, periostin acts to promote the collagen cross-linking in ECM. Interestingly, one splice variant $\left(\Delta \mathrm{b} \Delta \mathrm{e}\right.$ : deletion of exons $\mathrm{b}$ and $\mathrm{e}$ at the $3^{\prime}$ end of periostin mRNA) is preferentially expressed in response to $\operatorname{TGF} \beta$ at the beginning of regeneration of heart tissues and activates the integrin signals. This $\Delta \mathrm{b} \Delta \mathrm{e}$ variant form is also dominantly observed in the early stage of valve development [26] and in periodontal tissues in mice [35].

In the later chronic stage of a myocardial infarction, fibrillogenesis is accelerated by periostin and proceeds to generate a tight scar, in a similar fashion as in cardiac hypertrophy [17]. Furthermore, in an experiment in which rat cardiomyocytes were manipulated, Kuhn et al. [36] showed that periostin induced the proliferation of differentiated cardiomyocytes in the rat to improve ventricular remodeling and myocardial infarction. However, this proliferation was not observed in mice [37]. This discrepancy needs to be resolved in the future.

The regulatory hierarchies active in the development of cartilage, tendon, and bone are also important in heart valve maturation and ECM remodeling. In diseased heart valves, there is a distinct loss of ECM organization associated with changes in mechanical properties that ultimately lead to cardiac dysfunction [38]. In this respect, periostin is a good candidate to be tested for involvement in valve diseases. Hakuno et al. [26] reported that periostin enhances the degeneration of atherosclerotic and rheumatic cardiac valves. In mice fed with a high-fat diet, aortic valve thickening and annular fibrosis are increased, concomitant with increased periostin expression, which is correlated with tight scar formation after a myocardial infarction. Periostin enhances fibrillogenesis at the valve. Interestingly, this report additionally found a new periostin function, i.e., that periostin induces the secretion of MMP2 and MMP-9 from human epithelial cells and mouse macrophages, respectively. This finding suggests that periostin probably interacts with the precursor of MMP-2 or MMP-9 inside the cell for efficient secretion following proteolytic digestion to generate mature MMPs, which scenario would be consistent with Notch1 maturation by periostin after interaction of periostin and precursor Notch1 inside the cell [12].

Now, it is important to consider the two periostin functions, cell migration outside the cell and fibrillogenesis through protein association inside the cell, separately in heart diseases.

\section{Periostin in tumor fibrillogenesis}

Although other functions of periostin in tumorigenesis have been reviewed recently by Ruan et al. [19], I wish to address a new concept regarding periostin in tumor fibrillogenesis. During tumorigenesis, periostin is a potential contributor to tumor metastasis and epithelial-mesenchymal transition (EMT). In colon cancer, periostin is expressed in cancer-associated fibroblasts [39], and in pancreatic cancer, periostin is deposited in the periductal stroma [40]. In these cancers, however, periostin expression is not observed in the cancer cells themselves.

In contrast, periostin plays a role as a suppressor of invasion and metastasis in the progression of human bladder cancers [41]. Our observations on cancer cells in periostin $-/-$ mice show the importance of endogenous periostin in suppressing tumor size through activation of fibroblasts that produce ECM molecules for capsule formation [42]. By considering different actions in tumor malignancy, periostin-promoted cell migration or capsule formation suppressing tumor growth, we can precisely justify the periostin function in each tumor.

\section{Actions of the $\mathrm{C}$-terminal region of periostin}

Alternative splicing at the $3^{\prime}$ end of periostin generates 3 major variant forms: deletion of exon $b$, exon e, and exons $\mathrm{b}$ and $\mathrm{e}(\Delta \mathrm{b} \Delta \mathrm{e})$ in addition to the full form in mice. This splicing event at the $3^{\prime}$ end of periostin is evolutionarily 
conserved among mouse, human and zebrafish, suggesting an important function. The $\Delta \mathrm{b} \Delta \mathrm{e}$ variant of periostin is preferentially and commonly present in the periodontal ligament, periosteum, and heart tissues after a myocardial infarction. This form is also preferentially found in the corresponding human tissues (unpublished data). The major difference between the full form and the $\Delta b \Delta \mathrm{e}$ form of periostin is recognizable at the moment of their secretion. Although intact periostin is hardly secreted, the $\Delta \mathrm{b} \Delta \mathrm{e}$ form is effectively secreted, indicating that this variant form is preferentially localized at the outside of cells and on the ECM to interact with integrins. Myofibroblasts produce periostin, and they also express the splice variants of fibronectin and tenascin- $\mathrm{C}$ induced by the action of TGF $\beta$, suggesting the presence of a functional complex involving these three molecules. Han et al. [43] reported that TGF $\beta 1$ regulates fibronectin isoform expression and splicing factor SRp40 expression, suggesting the involvement of a common splicing system in the same cell and developmental stage that is organized by the same splicing factor.

Periostin protein is often cleaved by proteolysis in heart disease [15], periosteum [11], and during wound healing [44]. This cleavage occurs preferentially from the C-terminal end, although almost no functional observations regarding cleaved truncated periostins were reported. However, recently, we found that specific cleavage at a region near the C-terminus of periostin, which is a heparinbinding site [45], is essential for association of periostin with tenascin-C [11], indicating that proteolytic cleavage is functional in a developmental or pathological stagedependent manner. Further investigations regarding the function of proteolytic cleavage of periostin are required.

\section{Future perspective}

In Fig. 4, I have summarized a mechanistic view of periostin function. After inflammation or mechanical stress, the expression of TGF $\beta$ and/or IL-4 and IL-13 [9] is induced in macrophages and neutrophils (in response to inflammation) or in other types of cells (in response to mechanical stress). These cytokines trigger the expression of periostin as well as primarily splice variants of other ECM molecules (fibronectin and tenascin-C) in fibroblasts, by acting through transcription factors such as twist and c-fos. The splice variant of periostin is secreted and localized outside the cell in the ECM, where it interacts with integrin $\alpha \mathrm{v} \beta 3$ on myofibroblasts to induce their cell migration through downstream signals involving Akt and FAK phosphorylation. These myofibroblasts produce type I collagen to repair tissues. In this collagen production, periostin together with tenascin- $\mathrm{C}$ inside the cell forms a
Mechanistic view of periostin functions

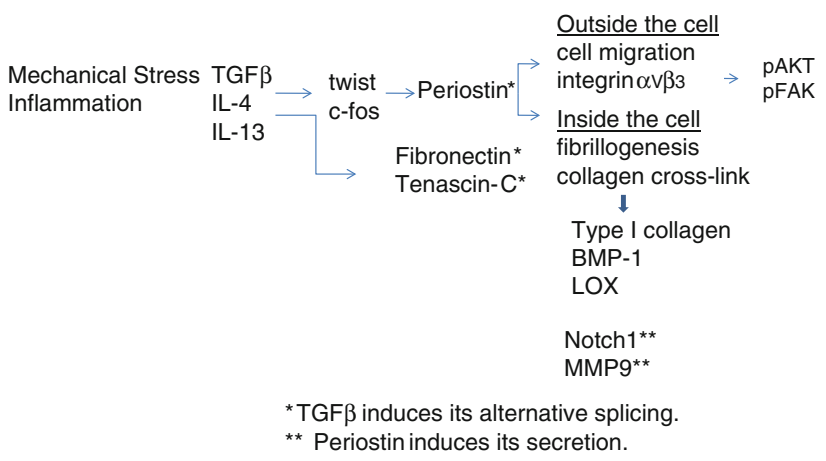

Fig. 4 Mechanistic view of periostin functions. Inflammation or mechanical stress induces the expression of TGF $\beta$ and/or IL-4 and IL13 in macrophages and neutrophils (for inflammation) or in other types of cells (for mechanical stress). These cytokines then induce the expression of periostin as well as that of other ECM molecules such as fibronectin and tenascin- $\mathrm{C}$ in fibroblasts, which proteins are mainly splice variants, by acting through transcription factors such as twist and c-fos. The splice variant of periostin is secreted and localized outside the cell in the ECM, where it interacts with integrin $\alpha \mathrm{v} \beta 3$ on myofibroblasts to induce their cell migration through downstream signals of Akt and FAK phosphorylation. These myofibroblasts produce type I collagen to repair tissues. In this collagen production, periostin together with tenascin- $\mathrm{C}$ inside the cell forms a meshwork structure with fibronectin to constitute a scaffold for the cross-linking of type I collagen. This cross-linking is effected by periostin in association with BMP-1 to activate lysyl oxidase (LOX) for enhancement of cross-linking activity inside the cells. During tissue repair, periostin helps in the secretion of MMP-9 and expression of Notch1 on the cell surface

meshwork structure with fibronectin to constitute a scaffold for the cross-linking of type I collagen. This cross-linking is effected by periostin in association with BMP-1 to activate lysyl oxidase for enhancement of cross-linking activity inside the cell. During tissue repair, periostin helps in the secretion of MMP-9 and expression of Notch1 on the cell surface, which activities are probably regulated by the association of precursor MMP-9 and Notch1 with periostin for effective proteolysis by a furin-like proteinase to generate their secreted mature forms.

Regarding extracellular collagen fibril growth after secretion of the type I collagen meshwork, the initial stage of ECM deposition results in arrays of narrow fibrils of uniform diameter, which diameter can increase by about tenfold. In the ECM, collagen fibril growth is thought to occur by accretion as well as by lateral and end-to-end fusion of collagen fibrils [33]. Thus, we need to further investigate the function of periostin in fibrillogenesis outside the cell and the new possible functions of periostin associated with matricellular proteins such as thrombospondins and CCN family members in the ECM. In our recent analysis, we found that periostin can associate with $\mathrm{CCN} 3$ to function in periodontal ligament (Tanabe et al., in preparation). Moreover, Hakuno et al. [26] have shown 
some interesting data indicating overlapping expression of periostin with elastin in the adult human cardiac valve, suggesting the involvement of periostin in elastin crosslinking outside the cell. Furthermore, in recent observations on periostin action outside the cell, periostin is found to associate with laminin on the basement membrane in hair follicles to induce keratinocyte proliferation during wound healing [44].

Finally, regarding pathological fibrillogenesis induced by periostin, over-protective reactions by periostin in pathogenesis accelerate fibrillogenesis, which gives rise to fibrosis. In an experimental model of lung fibrosis initiated using bleomycin, a high expression of periostin is induced, indicating a possible important action of periostin in tight collagen fiber formation (Kondo et al., unpublished data).

Acknowledgments I would like to thank the following collaborators at Tokyo Institute of Technology:Isao Kii, Masashi Shimazaki, Takashi Nishiyama, Takumi Maruhashi, Issei Takayama, and Hideyuki Tanabe. This work was supported by grants-in-aid for scientific research from the Ministry of Education, Science, Culture, and Sports of Japan and by grants from the Ground-based Research Program for Space Utilization promoted by Japan Space Forum.

Open Access This article is distributed under the terms of the Creative Commons Attribution Noncommercial License which permits any noncommercial use, distribution, and reproduction in any medium, provided the original author(s) and source are credited.

\section{References}

1. Horiuchi K, Amizuka N, Takeshita S, Takamatsu H, Katsuura M, Ozawa H, Toyama Y, Bonewald LF, Kudo A (1999) Identification and characterization of a novel protein, periostin with restricted expression to periosteum and periodontal ligament and increased expression by transforming growth factor $\beta$. J Bone Miner Res 14:1239-1249

2. Hortsh M, Goodman CS (1991) Cell and substrate adhesion molecules in Drosophia. Annu Rev Cell Biol 7:505-557

3. Kim JE, Kim SJ, Lee BH, Park RW, Kim KS, Kim IS (2000) Identification of motifs for cell adhesion within the repeated domains of transforming growth factor-beta-induced gene, beta ig-h3. J Biol Chem 275:30907-30915

4. Politz O, Gratchev A, McCourt PA, Schledzewski K, Guillot P, Johansson S, Svineng G, Franke P, Kannicht C, Kzhyshkowska J, Longati P, Velten FW, Johansson S, Goerdt S (2002) Stabilin-1 and -2 constitute a novel family of fasciclin-like hyaluronan receptor homologues. Biochem J 362:155-164

5. Takeshita S, Kikuno R, Tezuka K, Amann E (1993) Osteoblastspecific factor 2: cloning of a putative bone adhesion protein with homology with the insect protein fasciclin I. Biochem J 294:271-274

6. Munier FL, Korvatska E, Djemai A, Le Paslier D, Zografos L, Pescia G, Schorderet DF (1997) Kerato-epithelin mutations in four 5q31-linked corneal dystrophies. Nat Genet 15:247-251

7. Doliana R, Bot S, Bonaldo P, Colombatti A (2000) EMI, a novel cysteine-rich domain of EMILINs and other extracellular proteins, interacts with the $\mathrm{gClq}$ domains and participates in multimerization. FEBS Lett 484:164-168
8. Kii I, Amizuka N, Minqi L, Kitajima S, Saga Y, Kudo A (2006) Periostin is an extracellular matrix protein required for eruption of incisors in mice. Biochem Biophys Res Com 342:766-772

9. Takayama G, Arima K, Kanaji T, Toda H, Shoji S, McKenzie AN, Nagai H, Hotokebuchi T, Izuhara K (2006) Periostin: a novel component of subepithelial fibrosis of bronchial asthma downstream of IL-4 and IL-13 signals. J Allergy Clin Immunol 118:98-104

10. Norris RA, Damon B, Mironov V, Kasyanov V, Ramamurthi A, Moreno-Rodriguez R, Trusk T, Potts JD, Goodwin RL, Davis J, Hoffman S, Wen X, Sugi Y, Kern CB, Mjaatvedt CH, Turner DK, Oka T, Conway SJ, Molkentin JD, Forgacs G, Markwald RR (2007) Periostin regulates collagen fibrillogenesis and the biomechanical properties of connective tissues. J Cell Biochem 101:695-711

11. Kii I, Nishiyama T, Li M, Matsumoto K, Saito M, Amizuka N, Kudo A (2010) Incorporation of tenascin-C into the extracellular matrix by periostin underlies an extracellular meshwork architecture. J Biol Chem 285:2028-2039

12. Tanabe H, Takayama I, Nishiyama T, Simazaki M, Kii I, Li M, Amizuka N, Katsube K, Kudo A (2010) Periostin associates with Notch1 precursor to maintain Notch1 expression under a stress condition in mouse cells. PLoS ONE 5:e12234

13. Maruhashi T, Kii I, Saito M, Kudo A (2010) Interaction between periostin and BMP-1 promotes proteolytic activation of lysyl oxidase. J Biol Chem 285:13294-13303

14. Gillan L, Matei D, Fishman DA, Gerbin CS, Karlan BY, Chang DD (2002) Periostin secreted by epithelial ovarian carcinoma is a ligand for alpha $(\mathrm{V})$ beta $(3)$ and alpha $(\mathrm{V})$ beta (5) integrins and promotes cell motility. Cancer Res 62:5358-5364

15. Shimazaki M, Nakamura K, Kii I, Kashima T, Amizuka N, Li M, Saito M, Fukuda K, Nishiyama T, Kitajima S, Saga Y, Fukayama M, Sata M, Kudo A (2008) Periostin is essential for cardiac healing after acute myocardial infarction. J Exp Med 205:295-303

16. Rios H, Koushik SV, Wang H, Wang J, Zhou HM, Lindsley A, Rogers R, Chen Z, Maeda M, Kruzynska-Frejtag A, Feng JQ, Conway SJ (2005) Periostin null mice exhibit dwarfism, incisor enamel defects, and an early-onset periodontal disease-like phenotype. Mol Cell Biol 25:11131-11144

17. Oka T, Xu J, Kaiser RA, Melendez J, Hambleton M, Sargent MA, Lorts A, Brunskill EW, Dorn GW II, Conway SJ, Aronow BJ, Robbins J, Molkentin JD (2007) Genetic manipulation of periostin expression reveals a role in cardiac hypertrophy and ventricular remodeling. Circ Res 101:313-321

18. Norris RA, Moreno-Rodriguez R, Hoffman S, Markwald RR (2009) The many facets of the matricellular protein periostin during cardiac development, remodeling, and pathophysiology. J Cell Commun Signal 3:275-286

19. Ruan K, Bao S, Ouyang G (2009) The multifaceted role of periostin in tumorigenesis. Cell Mol Life Sci. doi:10.1007/ s00018-009-0013-7

20. Hamilton DW (2008) Functional role of periostin in development and wound repair: implications for connective tissue disease. J Cell Commun Signal 2:9-17

21. Dwek JR (2010) The periosteum: what is it, where is it, and what mimics it in its absence? Skeletal Radiol 39:319-323

22. Canty EG, Kadler KE (2005) Procollagen trafficking, processing and fibrillogenesis. J Cell Sci 118:1341-1353

23. Chiquet M, Gelman L, Lutz R, Maier S (2009) From mechanotransduction to extracellular matrix gene expression in fibroblasts. Biochem Biophys Acta 1793:911-920

24. Suzuki H, Amizuka N, Kii I, Kawano Y, Nozawa-Inoue K, Suzuki A, Yoshie H, Kudo A, Maeda T (2004) Immunohistochemical localization of periostin in tooth and its surrounding tissues in mouse mandibles during development. Anat Rec A 281:1264-1275 
25. Rios HF, Ma D, Xie Y, Giannobile WV, Bonewald LF, Conway SJ, Feng JQ (2008) Periostin is essential for the integrity and function of the periodontal ligament during occlusal loading in mice. J Periodontol 79:1480-1490

26. Hakuno D, Kimura N, Yoshioka M, Mukai M, Kimura T, Okada Y, Yozu R, Shukunami C, Hiraki Y, Kudo A, Ogawa S, Fukuda K (2010) Periostin advances atherosclerotic and rheumatic cardiac valve degeneration by inducing angiogenesis and MMP production in humans and rodents. J Clin Invest 120:2292-2306

27. Oshima A, Tanabe H, Yan T, Lowe GN, Glackin CA, Kudo A (2002) A novel mechanism for the regulation of osteoblast differentiation: transcription of periostin, a member of the fasciclin I family, is regulated by the bHLH transcription factor, twist. J Cell Biochem 86:792-804

28. Wilde J, Yokozeki M, Terai K, Kudo A, Moriyama K (2003) The divergent expression of periostin mRNA in the periodontal ligament during experimental tooth movement. Cell Tissue Res 312:345-351

29. Afanador E, Yokozeki M, Oba Y, Kitase Y, Takahashi T, Kudo A, Moriyama K (2005) Messenger RNA expression of periostin and twist transiently decrease by occlusal hypofunction in mouse periodontal ligament. Arch Oral Biol 50:1023-1031

30. Kashima TG, Nishiyama T, Shimazu K, Shimazaki M, Kii I, Grigoriadis AE, Fukayama F, Kudo A (2009) Periostin, a novel marker of intramembranous ossification, is expressed in fibrous dysplasia and in c-Fos-overexpressing bone lesions. Hum Pathol 40:226-237

31. Hirose Y, Suzuki H, Amizuka N, Shimomura J, Kawano Y, Nozawa-Inoue K, Kudo A, Maeda T (2003) Immunohistochemical localization of periostin in developing long bones of mice. Biomedical Res 24:31-37

32. Kudo H, Amizuka N, Araki K, Inohaya K, Kudo A (2004) Zebrafish periostin is required for the adhesion of muscle fiber bundles to the myoseptum and for the differentiation of muscle fibers. Dev Biol 267:473-487

33. Birk DE, Trelstad RL (1986) Extracellular compartments in tendon morphogenesis: collagen fibril, bundle, and macroaggregate formation. J Cell Biol 103:231-240

34. Bonnet N, Standley KN, Bianchi EN, Stadelmann V, Foti M, Conway SJ, Ferrari SL (2009) The matricellular protein periostin is required for Sost inhibition and the anabolic response to mechanical loading and physical activity. J Biol Chem 284:35939-35950
35. Takayama I, Kii I, Kudo A (2009) Expression, purification and characterization of soluble recombinant periostin protein produced by Escherichia coli. J Biochem 146:713-723

36. Kuhn B, del Monte F, Hajjar RJ, Chang Y-S, Lebeche D, Arab S, Keating MT (2007) Periostin induces proliferation of differentiated cardiomyocytes and promotes cardiac repair. Nat Med 13:962-969

37. Lorts A, Schwanekamp JA, Elrod JW, Sargent MA, Molkentin JD (2009) Genetic manipulation of periostin expression in the heart does not affect myocyte content, cell cycle activity, or cardiac repair. Circ Res 104:e1-e7

38. Lincoln J, Lange AW, Yutzey KE (2006) Hearts and bones: shared regulatory mechanisms in heart valve, cartilage, tendon, and bone development. Dev Biol 294:292-302

39. Kikuchi Y, Kashima TG, Nishiyama T, Shimazu K, Morishita Y, Shimazaki M, Kii I, Horie H, Nagai H, Kudo A, Fukayama M (2008) Periostin is expressed in pericryptal fibroblasts and cancer-associated fibroblasts in the colon. J Histochem Cytochem 56:753-764

40. Fukushima N, Kikuchi Y, Nishiyama T, Kudo A, Fukayama M (2008) Periostin deposition in the stroma of invasive and intraductal neoplasms of the pancreas. Mod Pathol 21:1044-1053

41. Kim CJ, Yoshioka N, Tambe Y, Kushima R, Okada Y, Inoue H (2005) Periostin is down-regulated in high grade human bladder cancers and suppresses in vitro cell invasiveness and in vivo metastasis of cancer cells. Int J Cancer 117:51-58

42. Shimazaki M, Kudo A (2008) Impaired capsule formation of tumors in periostin-null mice. Biochem Biophys Res Com 367:736-742

43. Han F, Gilbert JR, Harrison G, Adams CS, Freeman T, Tao Z, Zaka R, Liang H, Williams C, Tuan RS, Norton PA, Hickok NJ (2007) Transforming growth factor-b1 regulates fibronectin isoform expression and splicing factor SRp40 expression during ATDC5 chondrogenic maturation. Exp Cell Res 313:1518-1532

44. Nishiyama T, Kii I, Kashima TG, Kikuchi Y, Ohazama A, Shimazaki M, Fukayama M, Kudo A (2011) Delayed reepithelialization in periostin-deficient mice during cutaneous wound healing. PLoS ONE 6:e18410

45. Sugiura T, Takamatsu H, Kudo A, Amann E (1995) Expression and characterization of murine osteoblast-specific factor 2 (OSF2) in a baculovirus expression system. Protein Expr Purif 6:305-311 